\title{
Der Einfluß von Hypophysenextrakt auf die Magensaftsekretion.
}

\author{
Von \\ W. R. Hess und R. Gundlach. \\ (Aus dem Physiologischen Institut der Universität Zürich.) \\ Mit 3 Textabbildungen.
}

(Eingegangen am 10. Juli 1920.)

Neben den bekannten motorischen Effekten wird bekanntlich dem Hypophysenextrakt auch ein sekretorischer Einfluß zugeschrieben, nämlich auf Milchdrüse ${ }^{1}$ ) und Niere ${ }^{2}$ ) im Sinne einer Förderung. Die Speichelsekretion, vorher chemisch oder elektrisch angeregt, soll durch Hypophysenextrakt eine Hemmung erfahren ${ }^{3}$ ). Über den Einfluß des Hypophysenextraktes auf andere Verdauungsdrüsen sind, abgesehen von einem nicht weiter verwendeten Einzelversuch $\mathrm{P}$ o piels k is ${ }^{4}$ ) mit unphysiologischer Dosis, unseres Wissens noch keine Untersuchungen angestellt worden. Die richtige Einschätzung der physiologischen Bedeutung der Hypophyse lassen die Wünschbarkeit einer Ergänzung in dieser Richtung als selbstverständlich erscheinen. Auch die häufige therapeutische Anwendung aus der Hypophyse hergestellter Organpräparate verlangt, daß wir darüber orientiert sind, ob und inwieweit bei deren Applikation mit sekretorischen Begleiterscheinungen von seiten des Magendarmtraktus zu rechnen ist. Denn es ist nicht von vornherein ausgeschlossen, daß in dieser Beziehung eine so prägnante Wirkung zustande kommt, daß diese für die Verwendung des Extraktes neue Indikationen oder Kontraindikationen begründet.

Dies sind die Gründe, welche uns veranlaßten, den in der vorangehenden Arbeit als Testobjekt benützten Nebenmagenhund für die Beantwortung der hier aufgeworfenen Frage zu benützen. Die Versuchsanordnung und die Versuchsbedingungen decken sich vollständig mit den bereits beschriebenen, weshalb auf jene Ausführungen verwiesen

1) Ott und Scott, The action of infundibulin upon the mammary secretion. Proc. Soc. for Exp. Biol. and Med. 1910, S. 48.

$\left.{ }^{2}\right)$ Schäfer und Herring, The actions of pituitary extracts upon the kidney. Phil. transact. ser. 199. 1907.

3) Solem and Lommen, Influence of the extract of the posterior lobe of the hypophysis upon the secretion of saliva. Amer. journ. of physiol. 38, 3, S. 339.

4) Popielski, $\beta$-Imidazolyläthylamin als mächtiger Erreger der Magendrüsen. Pflügers Arch. Bd. 178, S. 214. 
werden kann (S.125). Auch hier wurde das Experiment nach der Art der Applikation (intramuskulär und intravenös) und nach dem Zeitpunkt derselben in bezug auf die Sekretionsphase variiert. Entweder erfolgte die Beibringung des Mittels gleich mit dem Fressen oder erst nachdem die Sekretion bereits eine gewisse Zeit in Gang gekommen war. Schließlich wurde die Prüfung auch auf die Zeit der Sekretionsruhe verlegt.

Zur Anwendung kam „Pituglandol“ der Firma Hoffmann-La Roche. Es wurde jeweils $0,5 \mathrm{ccm}$ gegeben, $d . h$. entsprechend $0,1 \mathrm{~g}$ frische Drüse (Infundibularteil). Für die Bewertung des unter Wirkung des Hypophysenextraktes zustandegekommenen Sekretionsverlaufes können dieselben Testversuche herangezogen werden, die schon für die Adrenalinversuche verwendet worden sind, die sich auf S. 128 u. 129 reproduziert und diskutiert finden.

Tabelle I. Konzentrationen der freien HCl.

Erläuterungen wie zu Tabelle II der vorhergehenden Arbeit betreffend Adrenalin.

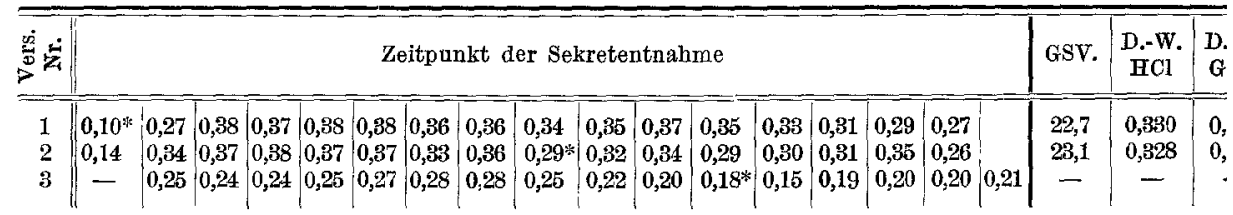

Über die Resultate der Versuche orientiert Tab. I. Sie führt die Daten auf, wie sie an dem viertelstündlich abgenommenen Sekret festgestellt worden sind in bezug auf Sekretvolumen, Kon-

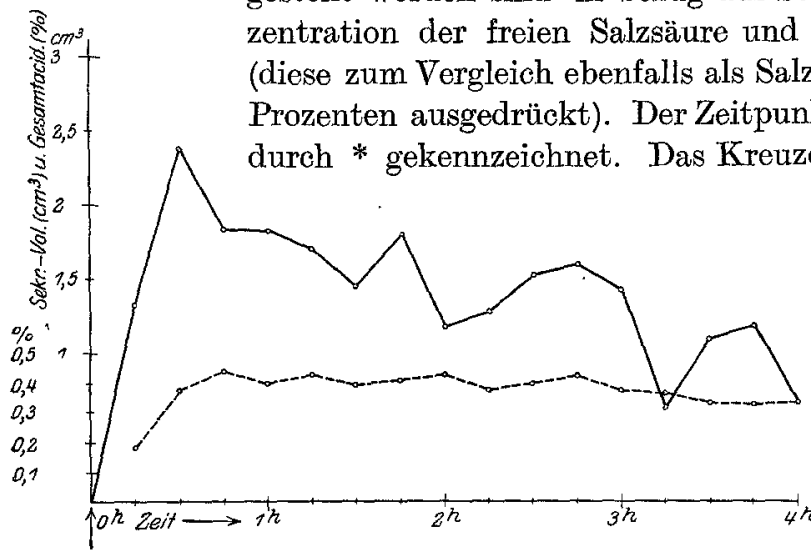

Abb. 1. Wirkung einer intramuskulären Pituglandolinjektion auf die Magensaftsekretion. —— Sekretvolumen. ........ Gesamt acidität. Die Injektion erfolgte bei $\uparrow, \mathbf{d} . \mathbf{h}$. während der Fưtterung. (Tab. I, Versuch Publ.-Nr, 1.). die sich auf das erste unter der Injektionswirkung zustandegekommene Sekret beziehen. Abb. 1 und 2 veranschaulichen den Sekretionsablauf in bezug auf Sekretvolumen und Gesamtacidität.

Die Durch prüf ang der Resultate ergibt, daß bei keiner unserer Untersuchungsserien, d. h. weder bei intravenöser noch bei intramuskulärer Applikation ein prägnanter Kinfluß zutage getreten ist. Wohl er- 
kennen wir dort, wo die Applikation nach bereits eingesetzter Sekretion erfolgte, eine kurz dauernde Depression an Sekretvolumina und Säurekonzentrationen. Das Defizit ist aber so gering, daß es mit der in der voranstehenden Arbeit beschriebenen Wirkung des psychischen Effektes in gleicher Ordnung steht und'als solche $\mathrm{zu}$ deuten ist ( $\mathrm{vgl}$. Tab. II und Abb. 6 auf S. 130). Durch diesen Befund wird der Erfolg der Adrenalininjektion unterstrichen, der zu einer quantitativ

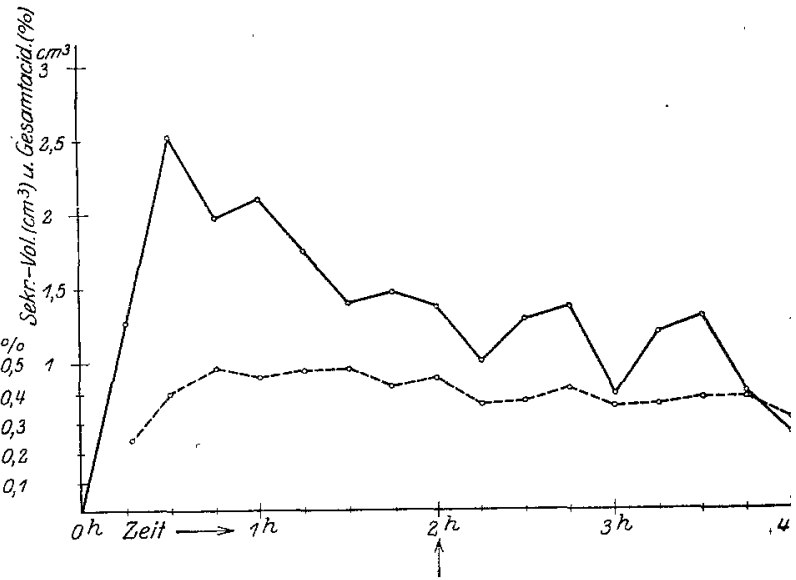

Abb. 2. Wirkung einer intramuskulären Adrenalininjektion in bezug auf die Magensaftsekretion. —_- Sekretvolumen. …..... Gesamtacidität. Die Injektion erfolgte bei $\uparrow$, d.h. 2 Stunden nach der Fütterung. (Tab. I, Versuch Publ.-Nr. 2.) und zeitlich weit intensiver ausgesprochenen Depression führt. Das Ausbleiben irgendeines deutlichen Effektes geht besonders schön aus Kurve 1 hervor. Diese selbst stimmt nämlich in größter Annäherung mit den Testkurven überein. Zum Vergleich kann mit Vorteil auch Abb. 2 dieser Publikation herangezogen werden, welche von dem Versuch des darauffolgenden Tages stammt. Bis nach Ablauf der zweitenSekretionsstunde ist der durch Kurve Nr. 2 illustrierte Sekretionsverlauf unbeeinflußt. Wir erkennen bei der ent-

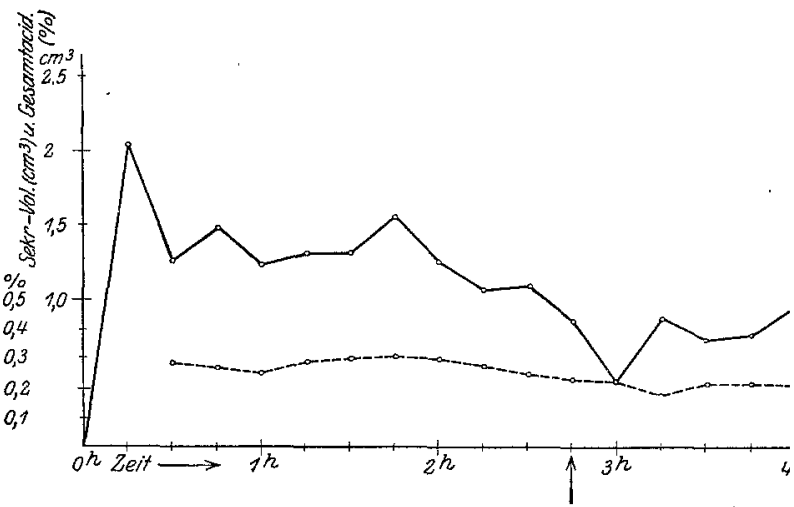

Abb. 8. Wirkung einer intravenösen Injektion von Pituglandol auf die Magensaftsekretion. ____- Sekretvolumen. -.....-.-Gesamtacidität. Die Injektion ins entnervte Ohr erfolgte bei $\uparrow$, d. h. $2^{3} / 4$ Stunden nach der Fïtterung. (Tab. I, Versuch Publ.-Nr. 3.) sprechenden Phase von Kurve Nr. 1 höchstens ein leichtes Zurückbleibe $\mathrm{n}$ der Sekretvolumina und der HCl-Konzentrationen, das man nicht mit Sicherheit als a ußerhalb der zufälligen Variation deuten kann. 
140 W. R. Hess und R. Gundlach: Der Einfluß von Hypophysenextrakt usw.

Zum Versuch Publ.-Nr. 3 (vgl. Tab I, Abb. 3) ist noch zu bemerken, daß die absoluten Werte wegen der auf S. 134 schon erwähnten Veränderung der Sekretionsbereitschaft des Magens, nicht mit den Testwerten verglichen werden dürfen. Das Resultat deckt sich vollständig mit dem schon beschriebenen, abgesehen von dem allgemeinen Tiefstand der Sekretion, welchen auch die Testversuche aus dieser späteren Zeit (Ende Mai) aufweisen. Die zwei von uns durchgeführten Versuchsreihen, bei welchen die intramuskuläre Verabreichung von Pituglandol in der Phase der Sekretionsr uhe erfolgte, verliefen ebenfalls negativ, $d . h$. ohne die Andeutung eines. Sekretionsreizes. Wir dürfen also auch nicht auf eine so geringfügige sekretionsfördernde Komponente schließen, daß sie in der Phase stärkerer Tätigkeit übersehen werden wäre.

Entsprechend der geführten Diskussion kommen wir zu dem Schluß, daß Hypophysenextrakt (Pituglandol) auf die Magensekretion höchstens einen ganz leicht depressorischen Effekt ausübt. Diese Erfahrung ist derjenigen gegenüberzustellen, welche Rothlin und Gundlach ${ }^{1}$ ) ebenfalls an unserem Hund mit Histamin machten. Die intramuskuläre Applikation dieser Substanz in einer Menge von 0,5 $\mathrm{mg}$ führt zu so profuser Sekretion, daß die gelegentlich schon vermutete Gegenwart von Histamin im Hypophysenextrakt in unseren Versuchen nicht hätte verborgen bleiben können; man müßte denn annehmen, daß andere Substanzen die Histaminwirkung kompensieren. Da diese Kompensation in jeder einzelnen Phase des ca. 60 Minuten dauernden Effektes eine quantitative sein müßte, ist deren Supposition nichts weniger als naheliegend.

\section{Nachtrag von W. R. Hess.}

Die vorliegenden zwei Arbeiten waren eben fertiggestellt, als der Mitarbeiter Reinhold Gundlach durch Unglück in den Bergen seinen Tod fand. Es ist uns dadurch ein angehender Physiologe von ungewöhnlichen Fähigkeiten verlorengegangen.

1) E. Rothlin und R. Gundlach, L'influence de l'Histamine sur la sécrétion gastrique. Journ. de Physiol. et de Pathol. génér. 1920. 